modalities can be exploited by utilising them in tandem. 4

OMP JOLOBE

Department of Medicine for the Elderly, Tameside General Hospital, Ashton-under-Lyne,

Lancashire OL6 9RW, UK

1 Yinnon AM, Hadas-Halpern I, Shapiro M, et al. The changing clinical spectrum of liver abscess: the Jerusalem experience. Postgrad Med f 1994; 70: 436-9.

2 Rubinson HA, Ishikoff MB, Hill, MC. Diagnostic imaging of hepatic abscess: a retrospective tic imaging of hepatic abscess: a
analysis. AfR 1980; 135: 735-40.

3 McDonald MI, Corey GR, Gallis HA, et al. McDonald MI, Corey GR, Gallis HA, et al.
Single and multiple pyogenic liver abscess. Single and multiple pyoge
Medicine 1984; 63: 291-302.

4 Shimada H, Ohta S, Mehara M, et al. Diagnostic and therapeutic strategies of pyogenic liver abscess. Int Surg 1993; 78: 40-5.

5 Barreda R, Ros PR. Diagnostic imaging of liver abscess. Crit Rev Diag Imaging 1992; 33: 29-58.

\section{Bone density measurement in osteogenesis imperfecta may well be important}

Sir

We thank Drs Deodhar and Woolf ${ }^{1}$ for their interest in our paper, ${ }^{2}$ We agree that bone densitometry may contribute to the diagnosis of osteogenesis imperfecta, a low finding being supportive of the diagnosis in some cases. However, in practice this is not often the problem; in most patients with clinical features or family history the diagnosis is not difficult without densitometry.

The practical difficulty is often the expectation that patients with osteogenesis imperfecta should have a low density as part of the disorder. Some do but in others the bone fragility must be largely attributable to the collagen disorder. Our paper should not be taken to suggest that patients with osteogenesis imperfecta with a bone density within the reference range do not have bone fragility. We were merely anxious to demonstrate that, since many patients had normal values on densitometry, such findings cannot be used to 'exclude' a diagnosis of osteogenesis imperfecta. Since the assessment of bone density by ordinary radiology is much less precise than any form of densitometry, ${ }^{3-5}$ still less is it possible to exclude osteogenesis imperfecta with plain films.

We disagree with Drs Deodhar and Woolf on the appropriateness of our methods. The great majority of known fractures in osteogenesis imperfecta are fractures of the long bones. Single photon absorptiometry may therefore be particularly appropriate in this disorder.

COLIN R PATERSON

PATRICIA A MOLE

Department of Biochemical Medicine, Ninewells Hospital and Medical School, Dundee DD1 9SY, Scotland, UK

1 Deodhar AA, Woolf AD. Bone density measurement in osteogenesis imperfecta may well be important. Postgrad Med $₹$ 1994; 70: 463-4.

2 Paterson CR, Mole PA. Bone density in osteogenesis imperfecta may well be normal. Postgrad Med F 1994; 70: 104-7.

3 Lachman E. Osteoporosis: the potentialities and limitations of its roentgenologic diagnosis. $A m \mathcal{F}$ Roentgenol 1995; 74: 712-5.

4 Finsen V, Anda S. Accuracy of visually estimated bone mineralization in routine radiographs of the lower extremity. Skeletal Radiol 1988; 17: 270-5.

5 Williamson MR, Boyd CM, Williamson SL. Osteoporosis: diagnosis by plain chest film versus dual photon densitometry. Skeletal Radiol 1990; 19: 27-30.

\section{Ileal leiomyoma - cause of undiagnosed chronic iron deficiency anaemia}

Sir,

Benign tumours of the small intestine are responsible for less than $1 \%$ of cases of gastrointestinal bleeding and hence present a daunting task in terms of diagnosis. ${ }^{1}$ Even after a thorough search, using all available diagnostic modalities, it may still be impossible to identify the source of chronic blood loss.

A 55-year-old Caucasian man was admitted with a two-day history of fever and lower abdominal pain. On examination he was found to be pyrexial and tachycardic with a tender mass in the left lower quadrant. The haemoglobin was $9.1 \mathrm{~g} / \mathrm{dl}$ with an iron deficiency picture and the white cell count was $13.3 \times 10^{9} / 1$. An ultrasound scan revealed $11 \times 10 \times 12 \mathrm{~cm}$ mass containing cystic elements arising posterior to the bladder. At operation a large pelvic mass arising from the ileum along with the enlarged mesenteric lymph nodes was found and these were excised en bloc with end-to-end anastomosis of the small bowel. The histology of the tumour revealed it to be a benign leiomyoma measuring $10 \times 11 \times 12 \mathrm{~cm}$, showing areas of acute inflammation and cystic degeneration. The enlarged mesenteric lymph nodes contain reactive changes only.

Looking back through the patient's case notes, we found that the patient had been thoroughly investigated 20 years ago for iron deficiency anaemia when no cause was found. He was again referred with the same problem three years ago when various investigations including gastroscopy, sigmoidoscopy, and large and small bowel enemas failed to reveal anything abnormal.

Despite the fact that the patient's haemoglobin had continued to fall over the past three years, he had refused further investigations.

The commonest benign tumours of the small intestine are adenomas, leiomyomas and lipomas. They may produce varying clinical pictures depending on the anatomical site, size and morphological characteristics (box). ${ }^{2}$ The present case is unusual because the tumour remained undetected having produced symptoms for 20 years. Contrast barium studies ${ }^{3}$ miss about $50 \%$ of these tumours, but enteroclysis ${ }^{4}$ is more sensitive in detecting the tumour and associated abnormalities, eg, thickening or separation of the bowel loops. The technique tests the distensibility of all small bowel segments, allows increased separation of small bowel loops with fewer missed lesions due to overlapping bowel, and has a double-contrast see-through effect for evaluating small bowel folds. Visceral angiography ${ }^{5}$ can demonstrate active bleeding, abnormal vasculature or ischaemia in a tumour, but the positive yield is extremely low either as a single study or as a repeat procedure; hence this investigation is superceded by a small bowel enema which can even detect subtle mucosal abnormalities. The role of enterscopy ${ }^{6}$ in chronic gastrointestinal bleeding of obscure origin is still a contentious issue because of the low diagnostic yield from 30 to $70 \%$ depending on the experience of the operator. CT $\operatorname{scan}^{7}$ is $97 \%$ sensitive in detecting small intestinal neoplasm. It can also detect adjacent and remote changes associated with the tumour, eg, enlarged mesenteric lymph nodes, thickened bowel wall, and liver metastases but it cannot
Benign tumours of the small intestine: clinical features

- intestinal obstruction ( $42 \%)$

- haemorrhage $(34 \%)$

- pain $(22 \%)$

- asymptomatic

detect active bleeding from the tumour. The role of the abdominal ultrasound is similar though it is less sensitive than CT.

Therefore, in conclusion we recommend that male and postmenopausal female patients with iron deficiency anaemia, in whom upper and lower alimentary endoscopy and small bowel barium studies are normal, should be further investigated by an abdominal CT scan with contrast and if this is negative, referred to a centre experienced in enteroscopy.

MUHAMMED ASHRAF MEMON ANTHONY R QUAYLE Department of Surgery, Macclesfield District General Hospital, Macclesfield, Cheshire, UK

Correspondence to Mr MA Memon, 10 Ridgemer Road, Pensby, Wirral, Merseyside L61 8RL, UK

1 Kaminski N, Shaham D, Eliakim R. Missed diagnosis: primary tumours of the duodenum. Postgrad Med f 1993; 69: 136-8.

2 Wilson JM, Melvin DB, Gray G, Thorbjarnarson B. Benign small bowel tumor. Ann Sur 1975; 181: 247-50.

3 Astley SW, Wells SA Jr. Tumors of the small intestine. Semin Oncol 1988; 15: 116-28.

4 Res DK, Lappas JC, Maglinte DD, Malczewski MC. Enteroclycis in the evaluation of suspected MC. Enteroclycis in the evaluation of suspected
small intestinal bleeding. Gastroenterology 1989 ; 97: 58 .

5 Lau WY, Ngan H, Chu KW, Yuen WK. Repea selective visceral angiography in patients with gastrointestinal bleeding of obscure origin. $\mathrm{Br}$ Surg 1989; 76: 226.

6 Schlauch D, Riemann JF. Enteroscopy - expansion of the diagnostic spectrum. Review and personal results. $Z$ Gastroenterol 1993; 31. 464-7.

7 Dudiak KM, Johnson CD, Stephens DH evaluation. $A f R$ 1989; 152: 995.

\section{Gestational macromastia not responding to termination of pregnancy}

Sir,

In a non-obese female, macromastia has been referred to as massive enlargement of breasts which cause discomfort to the patient because of their weight and is associated with crusting and ulcer formation on the breasts. Rarely, it can occur with pregnancy (gestational macromastia).

A 35-year-old married female during her second pregnancy noticed progressive enlargement of both breasts from the tenth week onwards, the breasts becoming massive in the next few weeks. Examination revealed an emaciated patient with mild hirsutism (familial) and pallor. Abdomen examination revealed a uterine height of 16 weeks. Breast examination revealed bilateral enlargement with diffuse tenderness, crusting, and superficial ulcers on the right. Investigations revealed haemoglobin of $6.9 \mathrm{~g} / \mathrm{dl}$, bone marrow aspirate revealed evidence of dimorphic anaemia (nutritional). Ultrasonography of ) 